\title{
Baekdusan Volcano Time-Series Analysis from 1992 to 1998 Using Multi-Interferogram InSAR Processing
}

\author{
Chang-Wook Lee* \\ National Institute of Meteorological Research, Korea Meteorological Administration, Jeju-do, Republic of Korea
}

Received 27 February 2014, revised 25 May 2014, accepted 6 June 2014

\begin{abstract}
A multi-temporal SAR interferometry (InSAR) method was applied to a time-series analysis of the period from 1992 - 1998 to detect precursor volcanic activity at Baekdusan volcano, located on the border between China and North Korea. Permanent scatterers were selected for stable signal processing to monitor ground motion using the permanent scatterer InSAR (PSInSAR) technique, which generated a time-series surface deformation map using the refined small baseline subset (SBAS) InSAR method. The time-series analysis results were verified using a multi-track comparison technique for two different tracks (88/230 and 89/230). The time-series analysis of the two tracks represents an unaltered phase signal (mm scale) with time, except for periods with insufficient interferograms to make a precise time-series surface deformation map due to factors including atmospheric phase delay and other error effects (i.e., those that display a maximum value of the phase difference from -2.14 to $+2.64 \mathrm{~cm}$ ). These phase signals also have the Root Mean Square Error (RMSE) range from $0.32-2.56 \mathrm{~cm}$. No surface deformation was detected from the Japanese Earth Resources Satellites (JERS-1) synthetic aperture radar (SAR) data collected between 1992 - 1998 at the Baekdusan volcano, as monitored using volcanic surface movements with dynamic magma activity. No volcanic eruption precursor signals were demonstrated during the inactive period (1992 - 1998) prior to the active period (2002 - 2006) of this volcano.
\end{abstract}

Key words: Baekdusan volcano, InSAR, Time-series analysis, Surface deformation

Citation: Lee, C. W., 2014: Baekdusan volcano time-series analysis from 1992 to 1998 using multi-interferogram InSAR processing. Terr. Atmos. Ocean. Sci., 25, 743-754, doi: 10.3319/TAO.2014.06.06.01(T)

\section{INTRODUCTION}

Baekdusan (Changbaishan) Mountain (Fig. 1) is located between China and North Korea on the eastern margin of the Eurasian plate. It is one of the active volcanoes in East Asia and was the site of one of the largest explosive eruptions in the last 2000 years (Kelly and Sear 1984; Rampino and Self 1984; Self 2006; Self et al. 2008), known as the Millennium eruption, which was the second largest volcanic eruption in history (Hetland et al. 2004; Wei et al. 2013; Xu et al. 2013). Volcanic ash from this eruption event, which occurred about 1000 years ago, was reported as far away as the northern part of Japan (approximately $1200 \mathrm{~km}$ ). This large eruption generated a caldera lake $\sim 4.5 \mathrm{~km}$ in diameter and nearly $1 \mathrm{~km}$ in depth at the summit of the mountain, reflecting a Volcanic Explosivity Index of 7 (Oppenheimer 2011; Wei et al. 2013). The geological characteristics of the Baekdusan volcano

\footnotetext{
* Corresponding author

E-mail:cwlee@korea.kr
}

have two types of deposits, pumice fall out and ignimbrite produced by the strongest of the most recent eruptions (Horn and Schmincke 2000; Yin et al. 2012). This volcano has been continuously monitored using measuring surface deformation from precise leveling over decades and by the China Earthquake Administration using GPS data since 1999 (Xu et al. 2012). In particular, the early part of the observation period showed surface displacements by volcanic activity until the middle of the period from 2002 - 2006, which was characterized by surface inflation phenomena. There was no surface deformation from the GPS and precision leveling survey in 2000 - 2002 and 2006 - 2010. Those long-term observations were successfully performed during 12 years of surface deformation monitoring. However, supplemental information for the expansion period can improve volcanic activity detection (Lee et al. 2013). As a result, Baekdusan volcano activity was assessed using Japanese Earth Resources Satellites (JERS-1) synthetic aperture radar (SAR) images 
from 1992 - 1998 before setting up monitoring stations at the Changbaishan Volcano Observatory (CHVO) in 1999. SAR interferometry (InSAR) is a powerful and efficient technique for monitoring surface deformation over a wide area (Massonnet and Feigl 1998), including such deformation over a large remote volcanic area. JERS-1 InSAR measured surface displacements on the Baekdusan volcano inceptively using two- and three-pass InSAR methods without time-series analysis (Kim et al. 2001). Although the InSAR technique can detect obvious volcanic area deformation during a short time period before and after volcanic activity using a single interferogram (Massonnet et al. 1995; Lu et al. 2000), multiinterferograms can measure slow deformation by monitoring changes in magma chamber volume using long-term time-series InSAR analysis (Ferretti et al. 2001; Hopper et al. 2004, 2007; Chaussard et al. 2013; Lu and Dzurisin 2014). The Baekdusan volcano showed slow surface deformation of a few centimeters during the active period from 2002 - 2006 (Cui et al. 2007; Xu et al. 2012). Therefore, it is necessary to analyze time-series surface deformation at Baekdusan volcano using appropriate combinations of multiple interferograms before setting up any surveying equipment. For this study a time-series technique was adopted for monitoring long-term surface deformation using the small baseline subset (SBAS) method with JERS-1 SAR data from 1992 - 1998, which can detect very slow deformations with sub-centimeter accuracy in two-dimensional planes (Fig. 2). A multi-track comparison was conducted for verifying the time-series analysis using two different neighboring tracks.

\section{DATA}

JERS-1 SAR data were acquired from the Japan Aerospace Exploration Agency (JAXA) to generate a surface deformation map associated with the Baekdusan volcanic activity. For this study SAR data were chosen for every track covering the Baekdusan volcanic area and all available archival data without seasonality and weather conditions. Two datasets showed descending tracks (Fig. 3) between September 1992 and October 1998 surrounding the study location, with two different paths and the same row: 88/230 (Track A) and 89/230 (Track B) (Table 1).

\section{METHOD}

JERS-1 SAR data (L-band) presents very efficient wavelengths $(23.5 \mathrm{~cm})$ for measuring surface deformation (Tobita et al. 1998) in a volcanic area covered by heavy vegetation, light snow, or ice (Table 2). These 23 JERS-1 SAR data were used to generate 67 interferograms from Track A and 18 SAR data were also used to created 63 interferograms using from Track B (Table 1) over 6 years. Figures 4 and 5 represent the most highly coherent images surrounding the Baekdusan volcano, including Tracks A and B. Each inter- ferogram shows phase differences in many different places, particularly in the Baekdusan Mountain area. However, a phase difference on an interferogram does not mean wholesurface deformation. The phase information of an interferogram includes surface deformation, atmospheric effects, orbital error, topographic error, temporal decorrelation and noise factors, as shown in Eq. (1).

$$
\begin{aligned}
& \Delta \phi(x, r) \approx \frac{4 \pi}{\lambda}\left\{\Delta d(x, r)+\frac{B_{\perp}}{r \sin \theta} \Delta z_{\text {topo }}(x, r)\right\} \\
& +\Delta \phi_{\text {atmo }}(x, r)+\Delta \phi_{\text {orbit }}(x, r)+\Delta \phi_{\text {temp }}(x, r)+\Delta \phi_{n}(x, r)
\end{aligned}
$$

where $x$ is the azimuth, $r$ is the slant-range pixel coordinate, $\lambda$ is the wavelength, $B_{\perp}$ is the perpendicular baseline, $\theta$ is the SAR look angle, $\Delta d$ is the surface deformation, $\Delta z_{\text {topo }}$ is the topographic height error, $\Delta \phi_{\text {atmo }}$ is the atmospheric phase delay artifact, $\Delta \phi_{\text {orbit }}$ is the orbital error, $\Delta \phi_{\text {temp }}$ is the temporal decorrelation, and $\Delta \phi_{n}$ is the phase due to other noise sources (Berardino et al. 2002). Of these, the atmospheric effect with altitude on the InSAR image is more diverse than that with flatland according to weather conditions due to the phase delay phenomenon using differences in mountain height (Zebker et al. 1997; Hanssen 2001). For example, Fig. 4b shows a large phase difference with one of the shortest time periods, whereas Fig. $4 \mathrm{~g}$ shows a small phase difference with the largest time interval (around 4 years) covering the period in Fig. 4b. Figure 4b represents the atmospheric phase delay effect corresponding to the topographic height in the Baekdusan Mountain area. Moreover, there is no relationship between the time interval of each interferogram and the variation in phase values. In particular, Fig. 4b shows a phase difference opposite that of Fig. 4h using the same slave or master image (29 October 1996) corresponding to the Baekdusan volcano height. This must be an atmospheric phase delay phenomenon relative to topographic height, with different phase signal patterns representing an increase (Fig. 4h) or decrease (Fig. 4b) in phase value toward the top of the mountain. Concretely, atmospheric artifacts are limited to a few $\mathrm{cm}$ phase delay in the case of flat areas while this contribution can be measured by dozens of $\mathrm{cm}$ in areas of relief (Hooper et al. 2012). The interferograms in Fig. 5 show larger phase changes than do those in Fig. 4, even though both have similar locations and time interval interferometric pairs. This means that error factors could be included in the phase information with the interferograms shown in Fig. 5, except for surface deformation, which is primarily relevant for atmospheric effects.

\section{RESULTS}

\subsection{Permanent Scatterers Selection}

To accomplish time-series processing using multi-interferograms, it is necessary to insure stable scatterer points 


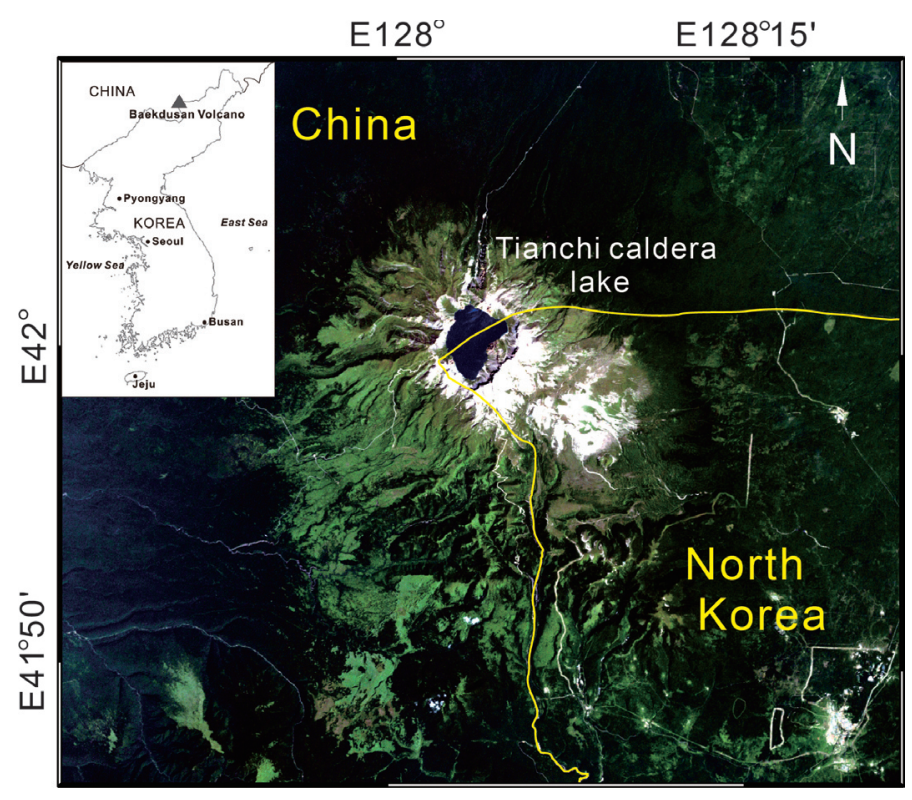

Fig. 1. LANDSAT-7 ETM+ image acquired on 25 August 2008, of Baekdusan volcano located at the border of China and North Korea. The blue lake represents the Tianchi volcanic caldera from the Millennium eruption, which is $5 \mathrm{~km}$ in diameter and $384 \mathrm{~m}$ deep. White color covering the mountain shows white pumice along the craggy cliffs near the caldera lake. The white pumice is especially widely distributed in the southeast region of the Tianchi crater.

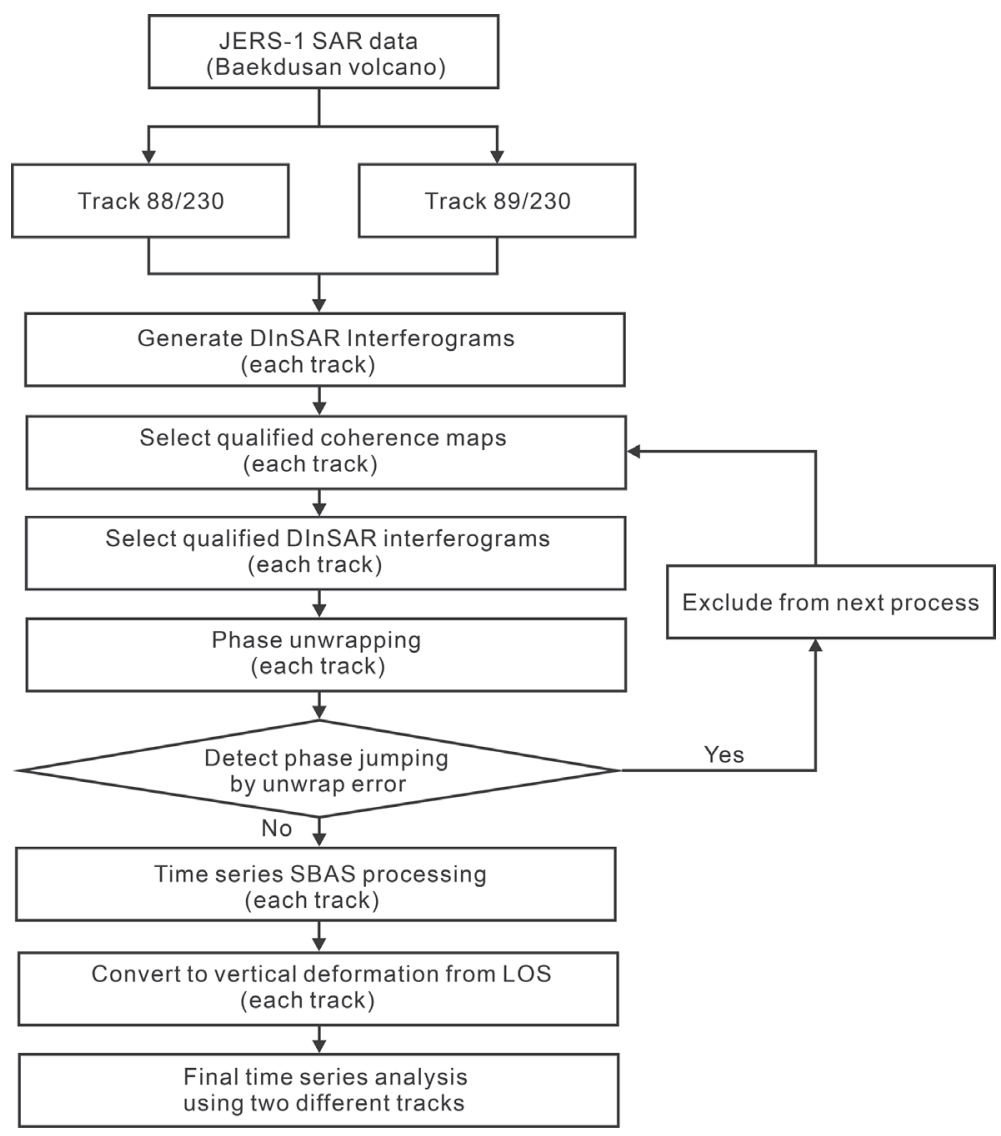

Fig. 2. Block diagram of this study using JERS-1 SAR data. 


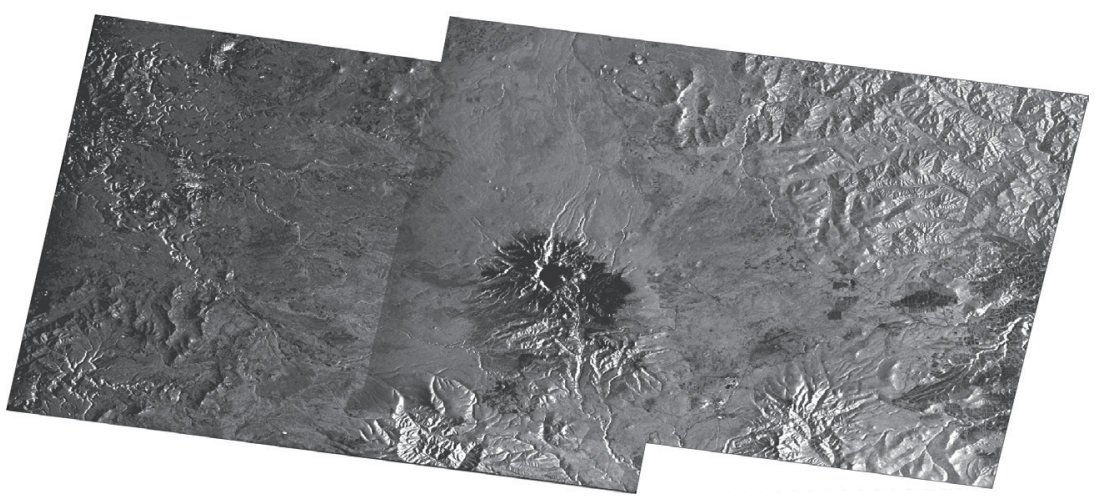

Fig. 3. Two independent overlapping tracks on the Baekdusan volcano using averaged JERS-1 SAR amplitude images. Image of track 88/230 is located on the right side, and track 89/230 is on the left side of Baekdusan Mountain.

Table 1. Two adjacent tracks on the Baekdusan volcano using averaged JERS-1 SAR images.

\begin{tabular}{|c|c|c|c|c|c|c|}
\hline \multicolumn{7}{|c|}{ JERS-1 SAR (Path/Row) } \\
\hline \multirow{2}{*}{ No. } & \multicolumn{3}{|c|}{ 88/230 (Track A) } & \multicolumn{3}{|c|}{ 89/230 (Track B) } \\
\hline & Date & Baseline (m) & Delta Days & Date & Baseline (m) & Delta Days \\
\hline 1 & $1992 / 09 / 24$ & 0 & 0 & $1992 / 09 / 25$ & 0 & 0 \\
\hline 2 & 1992/11/07 & 1362 & 44 & $1992 / 11 / 08$ & 1885 & 44 \\
\hline 3 & $1992 / 12 / 21$ & 1315 & 88 & $1992 / 12 / 22$ & 1601 & 88 \\
\hline 4 & 1993/02/03 & 4467 & 132 & $1993 / 06 / 16$ & 3559 & 264 \\
\hline 5 & 1993/03/19 & 2545 & 176 & $1994 / 01 / 22$ & 4554 & 484 \\
\hline 6 & $1993 / 05 / 02$ & 5330 & 220 & $1994 / 08 / 30$ & 650 & 704 \\
\hline 7 & $1993 / 06 / 15$ & 2253 & 264 & $1996 / 05 / 07$ & 4229 & 1320 \\
\hline 8 & $1994 / 01 / 21$ & 2869 & 484 & $1996 / 06 / 20$ & 3254 & 1364 \\
\hline 9 & $1994 / 08 / 29$ & -1525 & 704 & $1996 / 08 / 03$ & 3917 & 1408 \\
\hline 10 & $1995 / 02 / 21$ & 213 & 880 & $1996 / 10 / 30$ & 3580 & 1496 \\
\hline 11 & $1995 / 08 / 16$ & 165 & 1056 & $1996 / 12 / 13$ & 5056 & 1540 \\
\hline 12 & $1996 / 03 / 23$ & 1161 & 1276 & $1997 / 01 / 26$ & 4786 & 1584 \\
\hline 13 & $1996 / 08 / 02$ & 2292 & 1408 & $1997 / 10 / 17$ & -427 & 1848 \\
\hline 14 & $1996 / 10 / 29$ & 2117 & 1496 & $1998 / 04 / 11$ & 213 & 2024 \\
\hline 15 & $1996 / 12 / 12$ & 2767 & 1540 & $1998 / 05 / 25$ & 3650 & 2068 \\
\hline 16 & $1997 / 04 / 23$ & 3078 & 1672 & $1998 / 07 / 08$ & 479 & 2112 \\
\hline 17 & $1997 / 10 / 16$ & -500 & 1848 & $1998 / 08 / 21$ & 1591 & 2156 \\
\hline 18 & $1998 / 02 / 25$ & -233 & 1980 & $1998 / 10 / 04$ & 2187 & 2200 \\
\hline 19 & $1998 / 04 / 10$ & -383 & 2024 & & & \\
\hline 20 & $1998 / 05 / 24$ & 2244 & 2068 & & & \\
\hline 21 & 1998/07/07 & -263 & 2112 & & & \\
\hline 22 & $1998 / 08 / 20$ & 537 & 2156 & & & \\
\hline 23 & $1998 / 10 / 03$ & 1024 & 2200 & & & \\
\hline
\end{tabular}


Table 2. Technical specification of JERS-1 SAR data.

\begin{tabular}{ll}
\hline \multicolumn{1}{c}{ Sensor complement of SAR } & Value of JERS-1 \\
\hline Frequency (GHz) & 1.274086 \\
Wavelength (m) & 0.2353 \\
Sampling frequency (Hz) & 17076000 \\
Pulse repetition frequency (Hz) & 1555.2001953 \\
Pulse width ( $\mu$ s) & 35.0 \\
Chirp slope (Hz s $\left.{ }^{-1}\right)$ & $-0.42757 \mathrm{e} 12$ \\
Range bandwidth (MHz) & 14.964950 \\
Single look range resolution (m) & 10.016487 \\
Single look range spacing (m) & 8.77818 \\
Ground range spacing (m) & 13.8 \\
Ground azimuth spacing (m) & 4.5 \\
Single look azimuth resolution (m) & 8 \\
Single look azimuth spacing (m) & 4.9 \\
Swath width (km) & 75 \\
Look angle (deg) & 35.21 \\
Center incidence angle (deg) & 39 \\
Data rate (Mbit s-1) & 60 \\
Polarization & $\mathrm{HH}$ \\
\hline
\end{tabular}

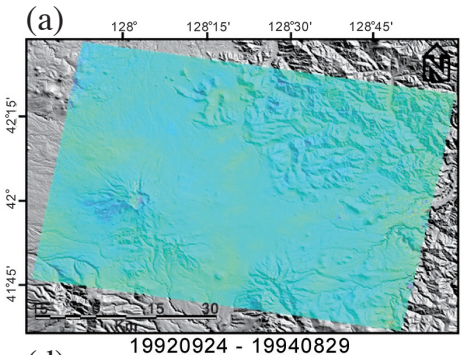

(d)

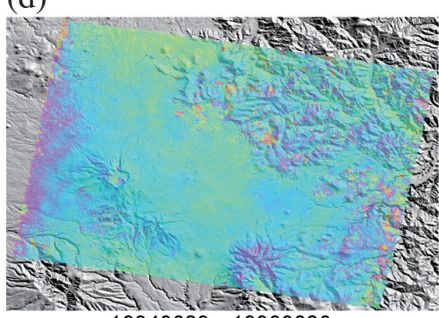

(g)

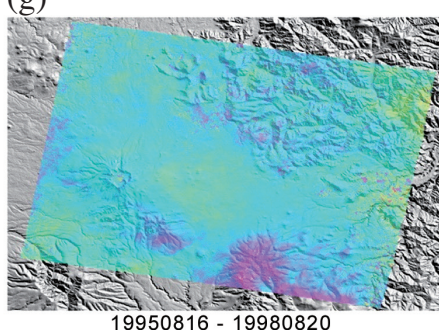

(b)

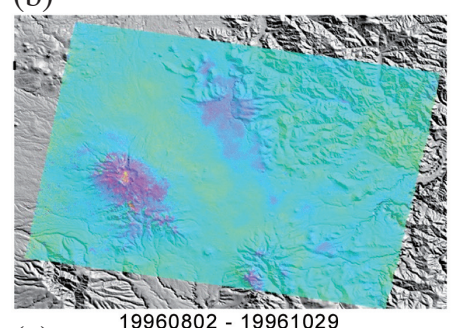

(e)

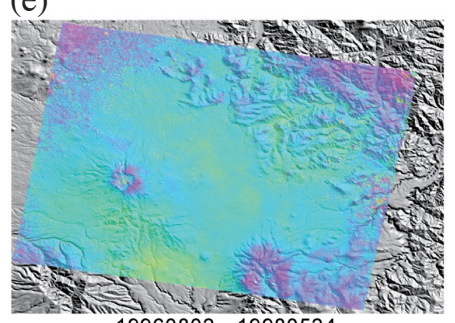

(h)

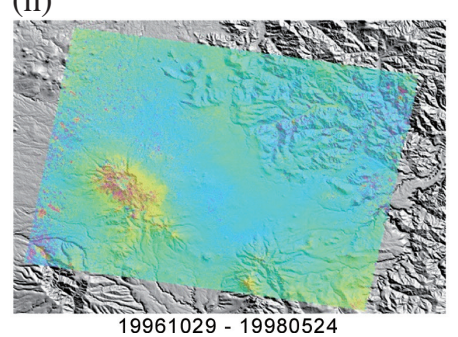

(c)

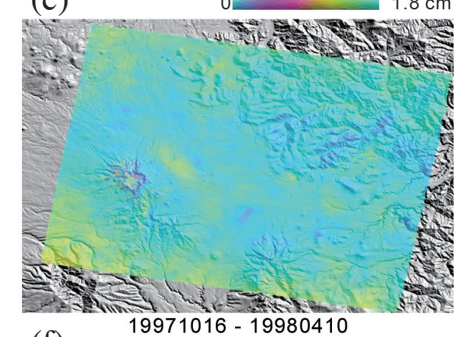

(f)

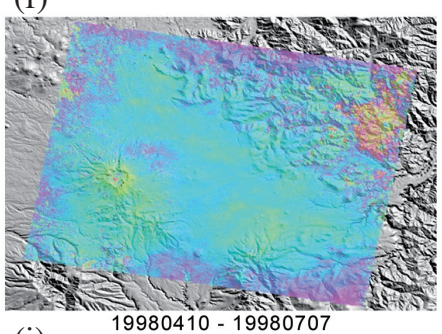

(i)

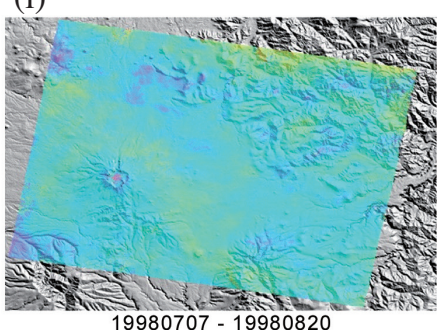

Fig. 4. High-coherent interferograms (a - i) for time-series SBAS processing from 1992 - 1998 for track 88/230. The color of these interferograms represents a change of phase difference in the direction of the line of sight. A color cycle of blue-green-yellow-red shows a shortening of the distance between the ground and the satellite. 
(a)

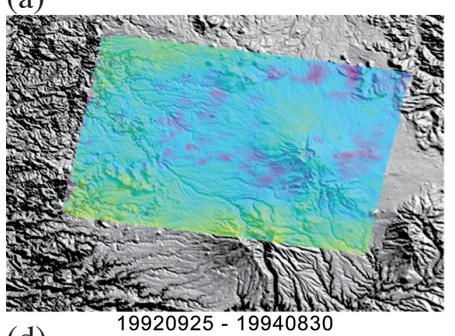

(d)

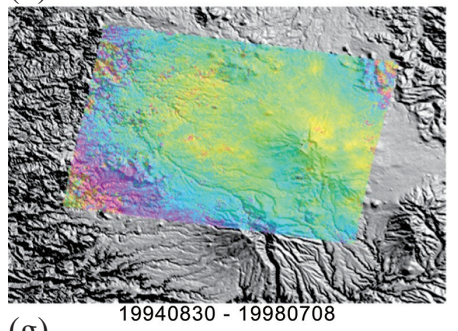

(g)

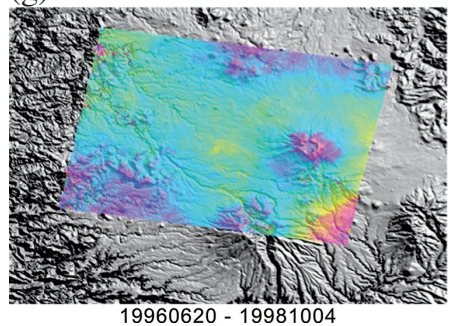

(b)

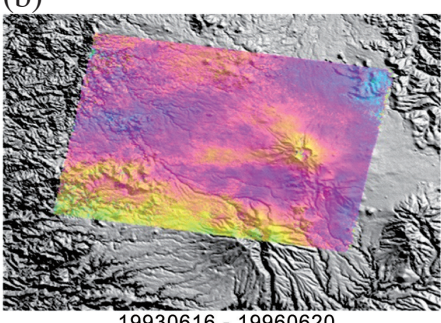

(e)

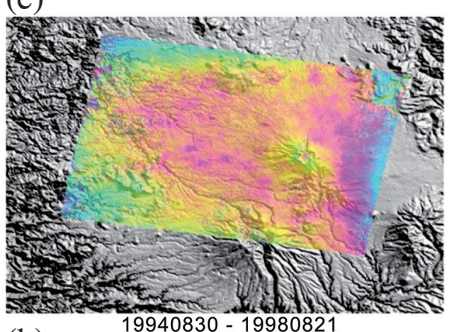

(h)

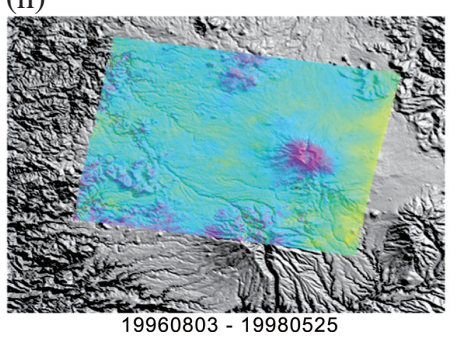

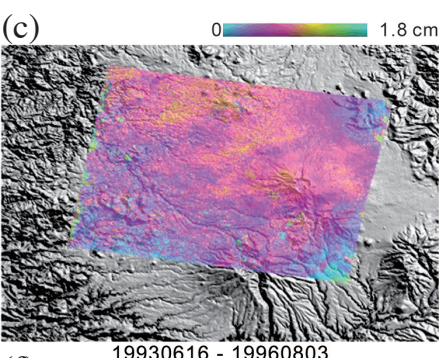

(f)

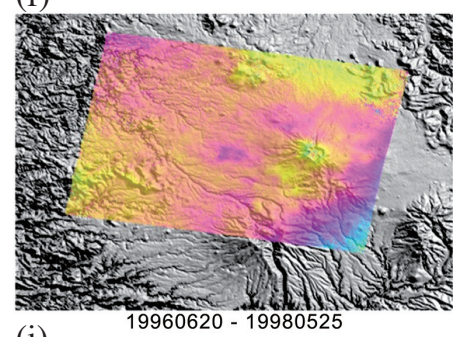

(i)

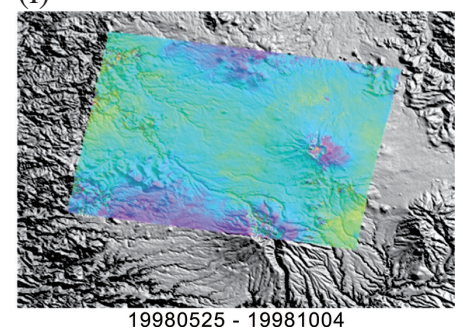

Fig. 5. High-coherent interferograms (a - i) for time series SBAS processing from $1992-1998$ for track 89/230. The color pattern shows a wider variety than that in Fig. 4 according to phase information such as surface deformation, atmospheric artifact effect, topographic height error, orbital error, and noise.

among entire pixels in the interferogram for all images used. Bounced satellite radar signals from permanent scattering points are collected by transmission towers, dams, bridges, rock outcrops, and other natural features consisting of prominent materials. The Baekdusan Mountain area is dominated by persistent natural features rather than artificial structures due to its remote location and distance from urban areas. Permanent scatterer points were extracted for the time-series processing from multiple master images (Zhang et al. 2011) using high-coherence pixels in every interferogram, such as the blue color pixels in Fig. 6.

\subsection{Time-Series Processing}

After selecting the permanent scatterer points the refined SBAS technique (Lee et al. 2010) is used to measure the surface displacements with time (Fig. 6). Only highcoherence interferograms are generally selected for timeseries analysis, as low-quality images for SBAS processing reduce coherent points in the interferogram and also produce incorrect phase information for making continuous time separations. The refined SBAS technique uses highcoherence images along with low-coherence images for a more detailed time-series analysis. This technique improves the surface deformation determination accuracy by reduc- ing error factors such as atmospheric artifacts, topographic errors, unwrapping errors and temporal noise through several iteration procedures (Lee et al. 2013). The atmospheric artifacts are reduced specifically by a temporal high-pass and spatial low-pass filtering procedure. Temporal decorrelation effects are mitigated by the finite difference smoothing approach. The orbital error phase bias is corrected using unwrapped residual interferograms calculated from the linear deformation rate (Lee et al. 2010). Twenty-five and 12 high-coherence images were selected together with 20 and 6 low-coherence images for each track, respectively (Fig. 7). Refined SBAS InSAR processing was applied to the Baekdusan volcano region to measure surface deformation using multiple InSAR data with time.

\subsection{Time-Series Analysis}

A mean surface deformation rate map (Fig. 8a) was generated for this large volcanic area using InSAR images taken between 1992 - 1998 for Track A (Table 1). Figure 8a shows similar phase values on every pixel in this map that is sky blue in color, except for some regions located in the western and eastern parts of the Baekdusan volcano that are pale yellow or blue in color. The phase value difference shows a maximum range of about $3 \mathrm{~cm}$ in the spatial domain, which 
is irregularly distributed in mountain and flatland areas and not located within a specific region according to the volcanic activity around the crater. This spatially scattered phase difference differs from the generalized surface deformation of the volcanic region. Thus, in addition to assessing spatial surface deformation, temporal analysis is required to determine more detailed fluctuations with time, including minute changes. Four different points (P1 - 4) were selected among the permanent scatterers in the map (Fig. 8a) to measure surface deformation between 1992 - 1998. Permanent scatterers around the crater should ideally be chosen to represent dynamic changes in the volcanic area. However, the area at the top of the mountain is usually covered in snow or ice due to the high elevation, creating generally low-coherence regions. Figure $8 b$ - e represents the time-series surface deformation according to each point around the Baekdusan volcano. Point P1 in Fig. 8a shows a phase difference with time near the crater and a clear inflation of about $7 \mathrm{~cm}$ between 2002 2005, as determined by field measurements (Xu et al. 2012). In contrast, no activity was indicated (Fig. 8b) during the 6 years before the active period (2002 - 2006). This fluctuation in signal is likely due to an atmospheric phase delay effect including other error factors rather than to surface deformation due to volcanic activity. No relationship was determined

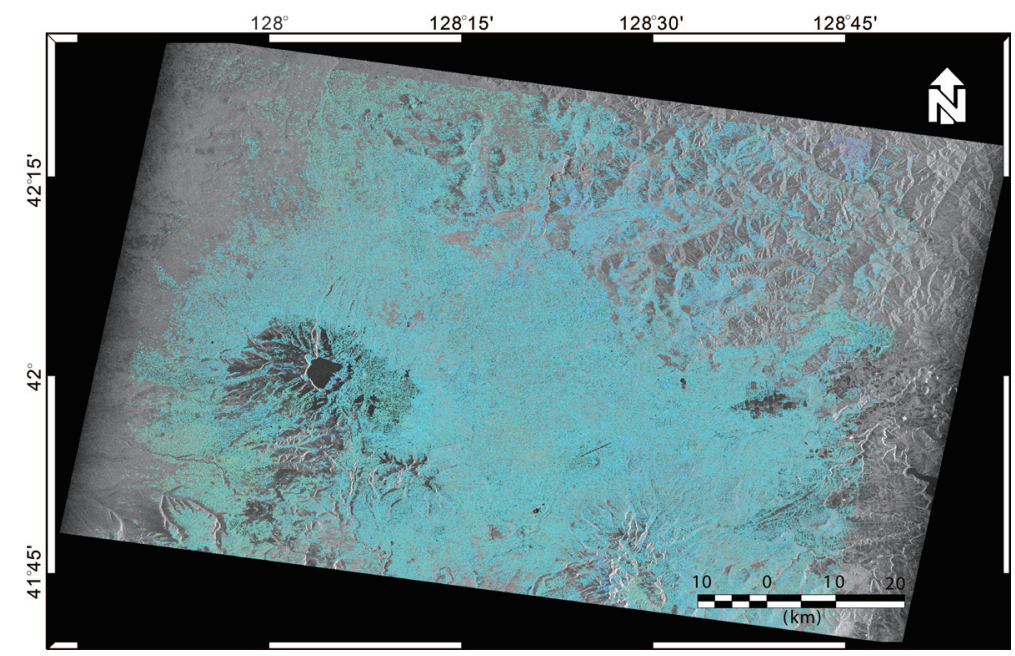

Fig. 6. Map of selected permanent scatterers in the Baekdusan volcano area from track 88/230 (1268195 points).

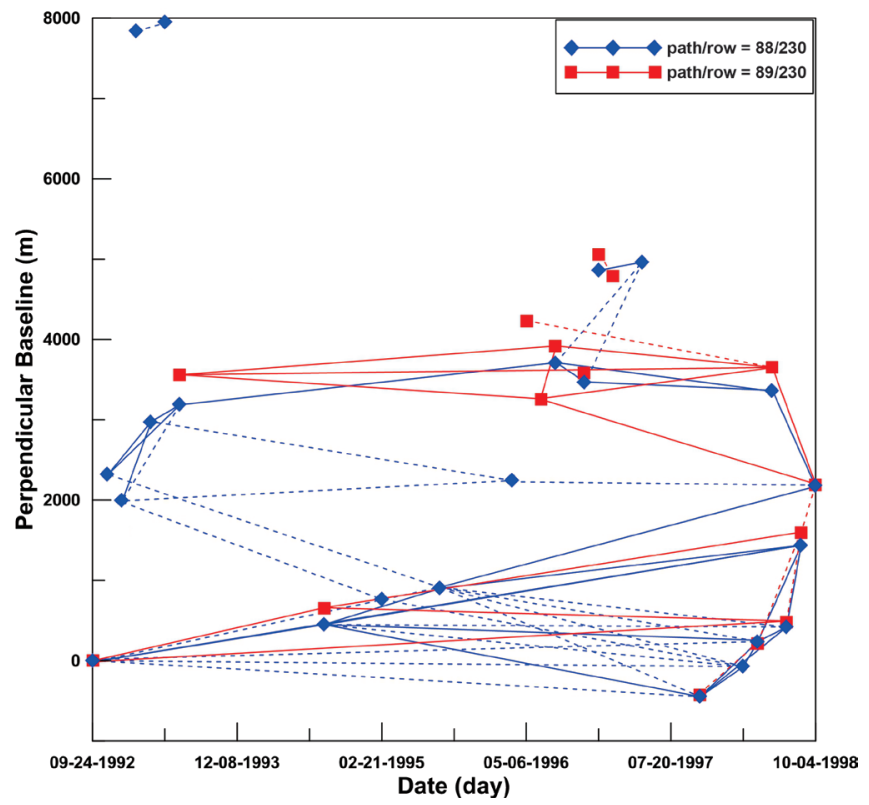

Fig. 7. Plots of perpendicular baseline and time for JERS-1 SAR dataset pairs available in the refined SBAS processing. Red squares and blue diamonds display the acquisition date of SAR data for tracks $88 / 230$ and $89 / 230$, respectively. The lines connecting the different dates indicate interferogram pairs (solid line: high-coherence interferograms, dotted line: low-coherence interferograms). 

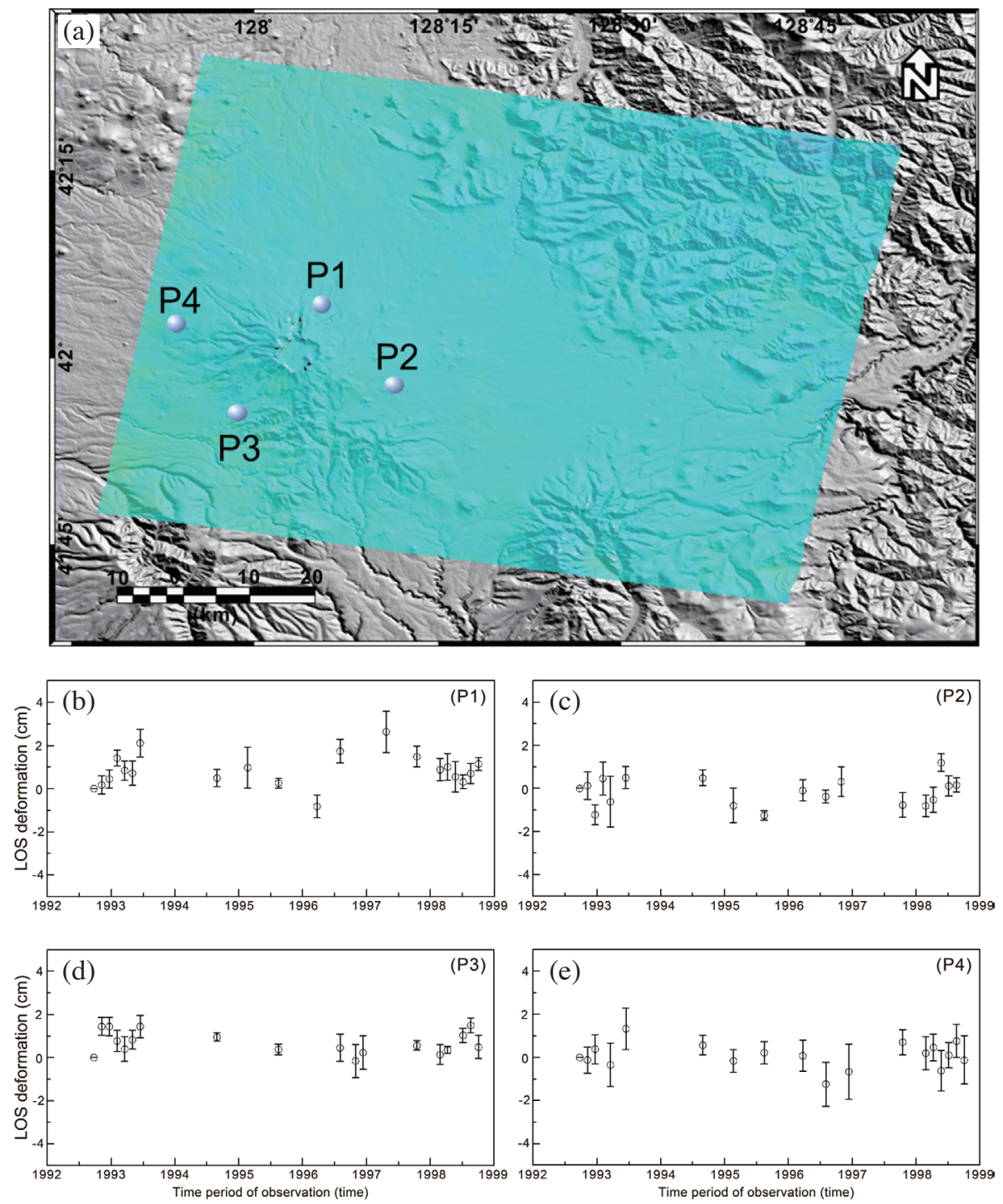

Fig. 8. Surface deformation velocity (a) and time-series distribution map (b - e) after refined SBAS processing for track 88/230. Error bars represent the range of the root mean squre error (RMSE) value for each point of the time-series phase difference during the 6 years.

between the phase difference and surface deformation through spatial and temporal signal analysis. The other three points, P2, 3, and 4 (Figs. 8c-e), also showed an oscillating signal for time-series between time intervals, with no indication of terrestrial movement. The data was processed for Track B (Table 1) for comparison with the time-series surface deformation analysis results because there are no in situ data with which to compare the refined SBAS result. Figure 9a shows the averaged surface deformation rate map using InSAR images for Track B after refined SBAS processing. In contrast to Fig. 8a, a blue color here indicates a higher elevation area and a yellow color indicates a low region along a drainage system. This phenomenon can be explained by an atmospheric phase delay effect with the mountain elevation, reflecting the amount of atmospheric phase between the interferograms in Figs. 4 and 5. Permanent scatterers were picked up for measuring time-series surface deformation in accordance with the four points in Fig. 8a. Large atmospheric phase delays also have an effect on time-series refined SBAS processing (Lee et al. 2012). Therefore, Figs. $9 \mathrm{~b}-\mathrm{e}$ show more atmospheric effects than do Figs. $8 \mathrm{~b}-\mathrm{e}$. No relationship with respect to time-series surface deformation was observed between Tracks A and B during the 6 years at the same permanent points. The direction along the line of sight of deformation $\phi_{\text {los }}$ can be converted into a vertical component $\phi_{v}$ for direct comparison according to different incidence angles $\phi_{i n c}$ from Figs. 8 - 9.

$\phi_{v}=\phi_{\text {los }} / \cos \left(\phi_{\text {inc }}\right)$

Figure 10 represents vertical surface movements between Tracks A and B based on refined SBAS results along 
permanent points such as P1 - 4. There was no correlation in terms of time-series surface deformation between the two tracks, with a scattered phase-change signal distribution of -2.14 to $+2.64 \mathrm{~cm}$ without constant inclination from Figs. $10 \mathrm{~b}$ - e. The best-fit lines (Figs. 10b - e) indicate time-series surface deformation using every time period point and combining the phase signal information of Tracks A and B.

\section{DISCUSSION}

Maximum vertical displacements of around $7 \mathrm{~cm}$ occurred at Baekdusan Mountain during the so-called "active volcanic period" between 2002 - 2006 (Ji et al. 2010; Xu et al. 2012; Ji et al. 2013). Surface movement measurements before the active period could provide useful insight for detecting impending volcanic activity. JERS-1 SAR data are the only method for detecting such surface deformation because of the lack of leveling or GPS surveys. Other SAR data with a different wavelength (C-band) are not efficient because of the large amount of vegetation and local temporal decorrelation such as ERS-1 and 2. A refined SBAS technique was proposed for multi-temporal InSAR analysis using two different tracks covering the Baekdusan volcanic area. Track A provided 25 high-quality interferograms from 6 years of data collection for generating a time-series analysis map, which is sufficient for refined SBAS processing. Therefore, the phase signal was generally stable with minor fluctuations, which elevated the reliability of the root mean square error range (RMSE) values from $0.32-2.56 \mathrm{~cm}$ in Fig. 8. Track B was processed with 12 interferograms, which is insufficient to interpret phase information from multitemporal InSAR analysis. The calculated RMS error value was between $0.33-2.38 \mathrm{~cm}$ using nine pixels based on permanent points such as P1 - 4 in Fig. 9. Tracks A and B were compared with each other directly through conversion into a direction vertical to the line of sight. The maximum phase
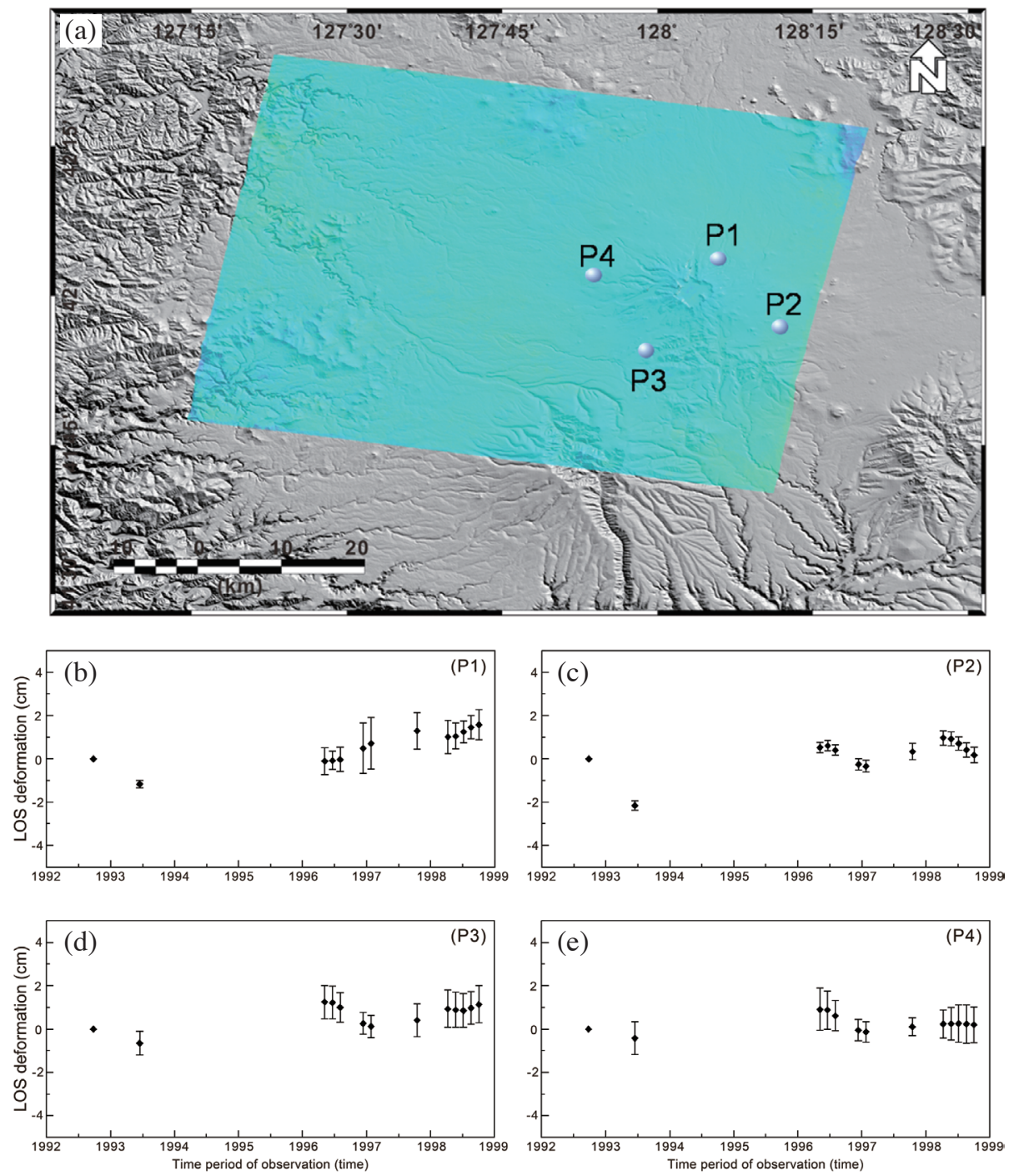

Fig. 9. Surface deformation velocity (a) and time-series distribution map (b - e) after refined SBAS processing for track 89/230. 

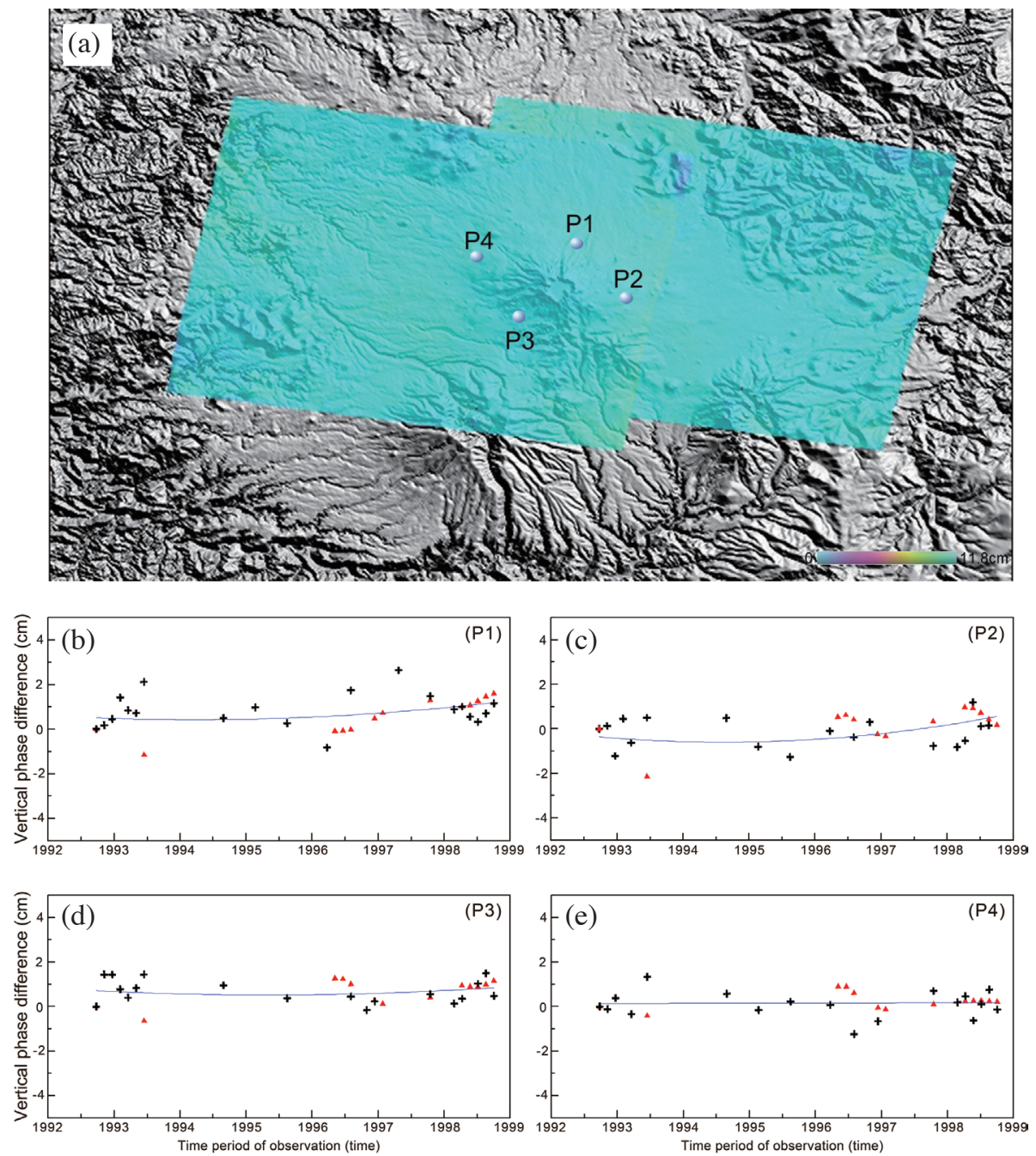

Fig. 10. Surface deformation velocity map with track A and B (a). Time-series surface deformation map (b - e) of vertical direction between tracks $88 / 230$ (black crosses) and 89/230 (red triangles). Blue line represents the second-order polynomial best fits for combining the time-series surface deformation of both tracks between 1992 - 1998.

difference between Tracks A and B was measured as about $4 \mathrm{~cm}$ on a time-series analysis near the middle of the period with a sparse time interval, whereas the minimum value of the phase difference represents the time around the end of the period with several millimeters using dense spacing between time gaps in Fig. 10. The best second-order polynomial lines, drawn with Tracks A and B to measure time-series surface deformation from 1992 - 1998 revealed slightly curved (Figs. 10b and c) or almost flat (Figs. 10d and e) properties. The time-series surface deformation or phase difference with time over 6 years had a reasonable and stable phase signal with slight fluctuations even though the interferograms showed an atmospheric phase delay phenomenon, including other error factors.

\section{CONCLUSIONS}

JERS-1 SAR data Multi-temporal InSAR processing for the Baekdusan volcano was performed to elucidate any precursor volcanic activity phenomena that occurred during the "active period" from 2002 - 2006 using leveling survey results. A refined SBAS method was used to generate an average surface deformation rate map for a two-dimensional spatial domain. Based on the permanent scatterers technique, four representative pixels $(\mathrm{P} 1$ - 4) were selected to measure the time-series surface deformation among the 1268195 persistent scatterers around the Baekdusan volcano area, as shown in Fig. 6. Point P1 in particular is located near the leveling survey area on the northeastern part of the volcano. A comparison of the multi-temporal InSAR results and field measurement data indicated that this area demonstrated the greatest surface inflation from $2002-2006$ (Xu et al. 2012; Ji et al. 2013). This can be verified by comparing the time-series analyses from two different tracks and converting the results into vertical direction displacements. The multi-track InSAR results with time indicated almost no 
surface deformation over 6 years, even though some phase difference fluctuations were present in the middle of the time domain due to atmospheric phase delay and other error factors, particularly the deficiency of interferogram pairs.

In summary, a refined SBAS time-series analysis using JERS-1 SAR data indicated that the Baekdusan volcano had an inactive period from 1992 - 1998 prior to the active period between 2002 - 2006. No precursor signal for the potential eruptive activity of this volcano was detected. Moreover, multi-sensor InSAR analysis can more precisely measure a time-series surface deformation in the Baekdusan volcano area by comparing field future measurement data.

Acknowledgements This research was supported by the National Institute of Meteorological Research (NIMR2012-B-3), Korea Meteorological Administration. SAR data in this study are copyrighted by the Alaska Satellite Facility. I thank the Editor-in-Chief and two Reviewers for their helpful comments and suggestions.

\section{REFERENCES}

Berardino, P., G. Fornaro, R. Lanari, and E. Sansosti, 2002: A new algorithm for surface deformation monitoring based on small baseline differential SAR interferograms. IEEE Trans. Geosci. Remote Sensing, 40, 23752383, doi: 10.1109/TGRS.2002.803792. [Link]

Chaussard, E., F. Amelung, and Y. Aoki, 2013: Characterization of open and closed volcanic systems in Indonesia and Mexico using InSAR time series. J. Geophys. Res., 118, 3957-3969, doi: 10.1002/jgrb.50288. [Link]

Cui, D. X., Q. L. Wang, K. Li, W. P. Wang, and Y. X. Hu, 2007: Analysis of recent deformation of Changbaishan Tianchi volcano. Chin. J. Geophys., 50, 1515-1524, doi: 10.1002/cjg2.1171. [Link]

Ferretti, A., C. Prati, and F. Rocca, 2001: Permanent scatterers in SAR interferometry. IEEE Trans. Geosci. Remote Sensing, 39, 8-20, doi: 10.1109/36.898661. [Link]

Hanssen, R. F., 2001: Radar Interferometry: Data Interpretation and Error Analysis, Remote Sensing and Digital Image Processing, Vol. 2, Springer, 308 pp.

Hetland, E. A., F. T. Wu, and J. L. Song, 2004: Crustal structure in the Changbaishan volcanic area, China, determined by modeling receiver functions. Tectonophysics, 386, 157-175, doi: 10.1016/j.tecto.2004.06.001. [Link]

Hooper, A., H. Zebker, P. Segall, and B. Kampes, 2004: A new method for measuring deformation on volcanoes and other natural terrains using InSAR persistent scatterers. Geophys. Res. Lett., 31, L23611, doi: 10.1029/2004GL021737. [Link]

Hooper, A., P. Segall, and H. Zebker, 2007: Persistent scatterer interferometric synthetic aperture radar for crustal deformation analysis, with application to Volcán Alcedo, Galápagos. J. Geophys. Res., 112, B07407, doi:

\subsection{9/2006JB004763. [Link]}

Hooper, A., D. Bekaert, K. Spaans, and M. Arikan, 2012: Recent advances in SAR interferometry time series analysis for measuring crustal deformation. Tectonophysics, 514517, 1-13, doi: 10.1016/j.tecto.2011.10.013. [Link]

Horn, S. and H. U. Schmincke, 2000: Volatile emission during the eruption of Baitoushan Volcano (China/North Korea) ca. 969 AD. Bull. Volcanol., 61, 537-555, doi: 10.1007/s004450050004. [Link]

Ji, L., J. Xu, X. Lin, and P. Luan, 2010: Application of satellite thermal infrared remote sensing in monitoring magmatic activity of Changbaishan Tianchi volcano. Chin. Sci. Bull., 55, 2731-2737, doi: 10.1007/s11434010-3232-2. [Link]

Ji, L., J. Xu, Q. Wang, and Y. Wan, 2013: Episodic deformation at Changbaishan Tianchi volcano, northeast China during 2004 to 2010, observed by persistent scatterer interferometric synthetic aperture radar. J. Appl. Remote Sens., 7, doi: 10.1117/1.JRS.7.073499. [Link]

Kelly, P. M. and C. B. Sear, 1984: Climatic impact of explosive volcanic eruptions. Nature, 311, 740-743, doi: 10.1038/311740a0. [Link]

Kim, S. W., J. S. Won, J. W. Kim, W. M. Moon, and K. D. Min, 2001: Multi temporal JERS-1 SAR investigation of Mt. Baekdu stratovolcano using differential interferometry. Geosci. J., 5, 301-312, doi: 10.1007/ BF02912701. [Link]

Lee, C. W., Z. Lu, H. S. Jung, J. S. Won, and D. Dzurisin, 2010: Surface deformation of Augustine Volcano, 1992-2005, from multiple-interferogram processing using a refined small baseline subset (SBAS) interferometric synthetic aperture radar (InSAR) approach. In: Power, J. A., M. L. Coombs, and J. T. Freymueller (Eds.), The 2006 Eruption of Augustine Volcano, Alaska: U.S. Geological Survey Professional Paper 1769, 453-465.

Lee, C. W., Z. Lu, and H. S. Jung, 2012: Simulation of timeseries surface deformation to validate a multi-interferogram InSAR processing technique. Int.J. Remote Sens., 33, 7075-7087, doi: 10.1080/01431161.2012.700137. [Link]

Lee, C. W., Z. Lu, J. S. Won, H. S. Jung, and D. Dzurisin, 2013: Dynamic deformation of Seguam Island, Alaska, 1992-2008, from multi-interferogram InSAR processing. J. Volcanol. Geotherm. Res., 260, 43-51, doi: 10.1016/j.jvolgeores.2013.05.009. [Link]

Lu, Z. and D. Dzurisin, 2014: InSAR imaging of Aleutian volcanoes. InSAR Imaging of Aleutian Volcanoes: Monitoring a Volcanic Arc from Space, Springer Praxis Books, Springer Berlin Heidelberg, 87-345, doi: 10.1007/978-3-642-00348-6_6. [Link]

Lu, Z., C. Wick Jr., J. A. Power, and D. Dzurisin, 2000: Ground deformation associated with the March 1996 earthquake swarm at Akutan volcano, Alaska, revealed 
by satellite radar interferometry. J. Geophys. Res., 105, 21483-21495, doi: 10.1029/2000JB900200. [Link]

Massonnet, D. and K. L. Feigl, 1998: Radar interferometry and its application to changes in the Earth's surface. Rev. Geophys., 36, 441-500, doi: 10.1029/97RG03139. [Link]

Massonnet, D., P. Briole, and A. Arnaud, 1995: Deflation of Mount Etna monitored by spaceborne radar interferometry. Nature, 375, 567-570, doi: 10.1038/375567a0. [Link]

Oppenheimer, C., 2011: Eruptions that Shook the World, Cambridge University Press, Cambridge, doi: 10.1017/ CBO9780511978012. [Link]

Rampino, M. R. and S. Self, 1984: Sulphur-rich volcanic eruptions and stratospheric aerosols. Nature, 310, 677679, doi: 10.1038/310677a0. [Link]

Self, S., 2006: The effects and consequences of very large explosive volcanic eruptions. Philos. T. R. Soc. A, 364, 2073-2097, doi: 10.1098/rsta.2006.1814. [Link]

Self, S., S. Blake, K. Sharma, M. Widdowson, and S. Sephton, 2008: Sulfur and chlorine in Late Cretaceous Deccan magmas and eruptive gas release. Science, 319, 1654-1657, doi: 10.1126/science.1152830. [Link]

Tobita, M., S. Fujiwara, S. Ozawa, P. A. Rosen, E. J. Fielding, C. L. Werner, M. Murakami, H. Nakagawa, K. Nitta, and M. Murakami, 1998: Deformation of the 1995 North Sakhalin earthquake detected by JERS-1/ SAR interferometry. Earth Planets Space, 50, 313325, doi: 10.1186/BF03352118. [Link]
Wei, H., G. Liu, and J. Gill, 2013: Review of eruptive activity at Tianchi volcano, Changbaishan, northeast China: Implications for possible future eruptions. Bull. Volcanol., 75, doi: 10.1007/s00445-013-0706-5. [Link]

Xu, J., G. Liu, J. Wu, Y. Ming, Q. Wang, D. Cui, Z. Shangguan, B. Pan, X. Lin, and J. Liu, 2012: Recent unrest of Changbaishan volcano, northeast China: A precursor of a future eruption? Geophys. Res. Lett., 39, L16305, doi: 10.1029/2012GL052600. [Link]

Xu, J., B. Pan, T. Liu, I. Hajdas, B. Zhao, H. Yu, R. Liu, and P. Zhao, 2013: Climatic impact of the Millennium eruption of Changbaishan volcano in China: New insights from high-precision radiocarbon wiggle-match dating. Geophys. Res. Lett., 40, 54-59, doi: 10.1029/2012GL054246. [Link]

Yin, J., A. J. T. Jull, G. S. Burr, and Y. Zheng, 2012: A wiggle-match age for the Millennium eruption of Tianchi Volcano at Changbaishan, Northeastern China. Quat. Sci. Rev., 47, 150-159, doi: 10.1016/j. quascirev.2012.05.015. [Link]

Zebker, H. A., P. A. Rosen, and S. Hensley, 1997: Atmospheric effects in interferometric synthetic aperture radar surface deformation and topographic maps. J. Geophys. Res., 102, 7547-7563, doi: 10.1029/96JB03804. [Link]

Zhang, L., X. Ding, and Z. Lu, 2011: Modeling PSInSAR time series without phase unwrapping. IEEE Trans. Geosci. Remote Sensing, 49, 547-556, doi: 10.1109/ TGRS.2010.2052625. [Link] 